\title{
Object Impedance Control Using a Closed-Chain Task Definition
}

\author{
Muhammad E. Abdallah, Charles W. Wampler, Robert Platt Jr.
}

\begin{abstract}
Humanoid robots are intended to interact with unstructured environments and to perform diverse applications. Often, such work involves manipulating an object cooperatively with multiple hands or fingers. This work presents an impedance based control framework for such cases with multipriority tasking. The primary task governs the impedance response of the object and a secondary task governs the impedance response of the joints. Using a novel transformation, the primary task may specify a subset of the object degrees of freedom (DOFs), allocating the remaining DOFs to the secondary task. This results in an integrated null space that includes not only the redundant DOFs of each manipulator independantly, but also the free DOFs of the object shared across the manipulators.
\end{abstract}

\section{INTRODUCTION}

The deployment of humanoid robots to manufacturing sites, especially assembly line work, requires robots to work in unstructured environments in which they physically interact with tools, surfaces, and disturbances while performing diverse tasks. Impedance control offers inherent advantages for such manipulation applications. First presented in [1], impedance control provides for robust interaction with the environment along with the flexibility to implement either force or motion objectives. These same advantages that arise in single-arm manipulation also apply to the cooperative control of an object using multiple manipulators.

This article presents an object impedance controller with two key features. First, it implements a multiple task hierarchy in which a second impedance relation operates in the redundant space of the first. The primary task applies to the operational space, while the secondary task applies to the joint space. The idea of prioritized multi-tasking is common in the area of motion control for manipulators [2]; however, its application to impedance control is new. This concept of multi-priority impedance was first presented in [3], where it was applied to serial-chain manipulation. This work extends the concept to cooperative control, where multiple serialchain manipulators close the loop on a single object.

Second, the primary task is defined on the object level to model a closed-chain mechanism. The primary task may thus specify impedances for only select degrees of freedom (DOFs) for the object, allocating the other DOFs to the secondary task. Hence, the null space of the primary task extends beyond the tip of the individual manipulator to include

M. Abdallah and C. Wampler are with the Manufacturing Systems Research Lab, General Motors R\&D, Warren, MI 48090, USA \{muhammad.abdallah, charles.w.wampler\}@gm.com. R. Platt was with the Johnson Space Center, NASA, Houston, TX 77058, USA robert.platt-1@nasa.gov.

Patents are pending on this work. the free DOFs of the object, a space now integrated across the manipulators. This paradigm facilitates both the primary and secondary tasks. For example, when the object's position is controlled but its orientation is free, the positional workspace is larger than it would be if orientation was also constrained. Moreover, the utilization of the extra freedoms can notably improve the fidelity of the secondary task, better enabling such features as joint-limit avoidance, obstacle avoidance, and null space damping. This closed-chain transformation presented here is applicable to other existing cooperative control frameworks as well.

Others have presented impedance laws for cooperative manipulation, but none with the key features presented here. In some cases, the laws govern the impedance of the manipulator end-effectors rather than the true impedance of the object [4], [5]. In other cases, the true object impedance is governed [6]; however, a dynamic model of the object and estimates of the acceleration are required-information that is inevitably noisy and inaccurate. This need for the object dynamics is eliminated in [7] by defining the impedance law with respect to a measure of internal force, but the external interaction of the object is no longer governed by a true impedance relation. Furthermore, [8] combines the two laws of [6] and [7] to control both the external and internal impedance of the object, but the law applies to non-redundant manipulators.

The formulation presented here applies a true object impedance. It eliminates the need for the object dynamics by introducing force feedback on the end-effectors. Such sensing is often accessible with higher fidelity than attempts to model the object and estimate its acceleration.

The controller is validated in simulation. A fully-dynamic model of a humanoid robot is created with two 7-DOF arms. The simulation demonstrates the performance of the multipriority impedance and closed-chain task definition.

\section{IMPEDANCE LAWS}

Our controller implements two impedance relations in a hierarchical framework. The primary impedance law dictates the behavior of the object and is defined by the following relation.

$$
\begin{gathered}
M_{o} \ddot{\boldsymbol{y}}+B_{o} \dot{\boldsymbol{y}}+K_{o} \Delta \boldsymbol{y}=\boldsymbol{F}-\boldsymbol{F}^{*} \\
\dot{\boldsymbol{y}} \doteq\left(\begin{array}{c}
\boldsymbol{v} \\
\boldsymbol{\omega}
\end{array}\right)
\end{gathered}
$$

In this expression, $M_{o}, B_{o}$, and $K_{o}$ are the commanded inertia, damping, and stiffness matrices respectively, where all are symmetric, $6 \times 6$ matrices. $\boldsymbol{v}$ is the linear velocity of the reference point on the object while $\boldsymbol{\omega}$ is the angular 
velocity of the object; both are measured with respect to the ground reference frame. $\boldsymbol{F}$ and $\boldsymbol{F}^{*}$ represent the net actual and desired external wrench, respectively, acting on the object. $\Delta \boldsymbol{y}$ is the position error, where the linear component is expressed by $\left(\boldsymbol{y}-\boldsymbol{y}^{*}\right)$ and the angular component is expressed in an angle-axis representation [9]. Note that the angular component is thus not a true integral of $\boldsymbol{\omega}$ despite the notation. Throughout this paper, bold symbols refer to either spatial vectors or column matrices.

At equilibrium, the external force $\boldsymbol{F}$ should be the sum of the nominal force $\boldsymbol{F}^{*}$ and the spring force $K_{o} \Delta \boldsymbol{y}$. If it is desired for some directions to be pure force control, this may be accomplished by setting the stiffness of those directions to zero in $K_{o}$. Given positive-definite matrices for $M_{o}, B_{o}$, and $K_{o}$, the impedance relation specifies a stable response.

The redundancy of the manipulators allows for a secondary task to act in the null space of the object impedance. We specify a joint space impedance law as:

$$
M_{j} \ddot{\boldsymbol{q}}+B_{j} \dot{\boldsymbol{q}}+K_{j} \Delta \boldsymbol{q}=\boldsymbol{\tau}_{f},
$$

where $M_{j}, B_{j}$, and $K_{j}$ are the commanded inertia, damping, and stiffness matrices, respectively, $\boldsymbol{q}$ is the column matrix of joint angles for all manipulators in the system, $\Delta \boldsymbol{q}$ is the joint position error, and $\tau_{f}$ represents the column matrix of joint torques produced by forces acting on the manipulator.

\section{KinEMATIC TRANSFORMATIONS}

To implement the object impedance task in (1), a kinematic transformation is needed to map the accelerations from the object space down to the joint space. The null space of this transformation is available for performing the secondary task. In the literature, such a transformation has been formulated by combining open-chain Jacobian matrices for each manipulator with a grasp matrix relating the velocity of each end-effector to the velocity of the object (both linear and angular). This tacitly assumes that all object degrees of freedom are specified in the primary task. To free up some object DOFs, we adopt a closed-chain perspective that collectively modifies the grasp and Jacobian transformations. We first review the open-chain transformations and then we combine them to form the closed-chain transformation for a reduced set of object DOFs.

\section{A. Open-Chain Kinematics}

The free-body diagram of the object and the coordinate system are shown in Fig. 1, where $N$ and $B$ represent the ground and body reference frames, respectively. Suppose that there are $n$ points of contact between the robot and the manipulated object. For $i=1, \ldots, n$, let $\boldsymbol{r}_{i}$ be the position vector from the reference point to the $i$ th contact point and let $\boldsymbol{v}_{i}$ and $\boldsymbol{\omega}_{i}$ be the velocity and angular velocity of an end-effector frame whose origin coincides with that point.

The acceleration of the body $B$ and the acceleration of the $i$ th contact frame of reference are related as:

$$
\begin{aligned}
& \dot{\boldsymbol{v}}_{i}=\dot{\boldsymbol{v}}+\dot{\boldsymbol{\omega}} \times \boldsymbol{r}_{i}+\boldsymbol{\omega} \times\left(\boldsymbol{\omega} \times \boldsymbol{r}_{i}\right)+2 \boldsymbol{\omega} \times \boldsymbol{v}_{\text {rel }_{i}}+\boldsymbol{a}_{r e l_{i}} \\
& \dot{\boldsymbol{\omega}}_{i}=\dot{\boldsymbol{\omega}}+\boldsymbol{\alpha}_{\text {rel }_{i}} .
\end{aligned}
$$

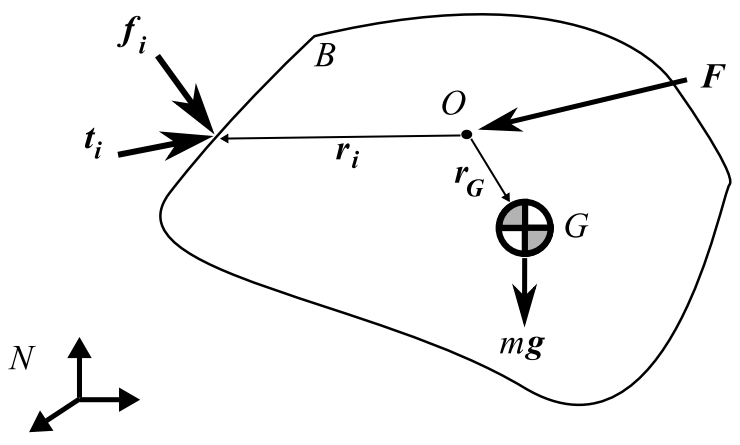

Fig. 1. Free-body diagram of object $B$, showing a force, $\boldsymbol{f}_{i}$, and a torque, $\boldsymbol{t}_{i}$, at contact point $i . O$ and $G$ represent the reference point and center of mass, respectively.

Here, $\boldsymbol{v}_{\text {rel }_{i}}$ and $\boldsymbol{a}_{r e l_{i}}$ are the first and second derivatives, respectively, of $\boldsymbol{r}_{i}$ in the object frame $B . \boldsymbol{\omega}_{\text {rel }_{i}}$ is the relative angular velocity between $B$ and the $i$ th end-effector frame, and $\boldsymbol{\alpha}_{r e l_{i}}$ is its time derivative in the ground frame. If the end-effectors are rigidly attached to $B$ and if the object is completely rigid, then all of the relative velocities and accelerations, i.e., $\boldsymbol{v}_{r e l_{i}}, \boldsymbol{a}_{r_{e l}}, \boldsymbol{\omega}_{r e l_{i}}$, and $\boldsymbol{\alpha}_{r e l_{i}}$, must be zero. In reality, the object and the end-effectors will have some compliance and relative motion between them that may be used to induce a desired change in internal forces, as we shall explore in a later section.

The acceleration relations (3) can be expressed in matrix form as the familiar grasp mapping. Let $\dot{\boldsymbol{x}}$ be a column matrix of all the velocities $\boldsymbol{v}_{i}$ and/or $\boldsymbol{\omega}_{i}$ that are constrained by the nature of the contact between the robot and the object, and let $\ddot{\boldsymbol{y}}$ be as in (1). Then,

$$
\ddot{\boldsymbol{x}}=G \ddot{\boldsymbol{y}}+\boldsymbol{h}
$$

where $G$ is known as the grasp matrix, and $\boldsymbol{h}$ is a column matrix of the relevant centripetal, Coriolis, and relative accelerations. The forms of $G$ and $\boldsymbol{h}$ depend on the grasp type, as we will see shortly.

While (4) summarizes the dependence of the contact frame accelerations, $\ddot{\boldsymbol{x}}$, on the object motion, $\ddot{\boldsymbol{y}}$, we may also use the forward kinematics of each branch of the manipulator to express $\ddot{\boldsymbol{x}}$ in terms of the joint velocities and accelerations, hence:

$$
\ddot{\boldsymbol{x}}=J \ddot{\boldsymbol{q}}+\dot{J} \dot{\boldsymbol{q}}=G \ddot{\boldsymbol{y}}+\boldsymbol{h},
$$

where $J$ is the column-wise concatenation of Jacobian matrices for the relevant velocities and/or angular velocities of the contact frames.

\section{B. Grasp Types}

In this transformation, the structures of $J, G$, and $\boldsymbol{h}$ depend on the grasp type. To illustrate, we will consider the following two grasp types.

- Two-Hand Grasp: A hand grasp represents a rigid contact that can transfer both arbitrary forces and moments, thus constraining both the linear and angular motion of the end-effector. Accordingly, for a two-hand grasp, $\dot{x}$ is a column matrix stacking the vectors $\boldsymbol{v}_{1}, \boldsymbol{\omega}_{1}, \boldsymbol{v}_{2}, \boldsymbol{\omega}_{2}$. 
- Three-Finger Grasp: If we model a finger contact as a no-slip, point contact, then it transmits only force and constrains just position. Accordingly, for a three-finger grasp, $\dot{\boldsymbol{x}}$ is a column matrix stacking just the linear velocities $\boldsymbol{v}_{1}, \boldsymbol{v}_{2}, \boldsymbol{v}_{3}$.

Referring to (3), the form of each row in $G$ corresponds to the entry type in $\dot{\boldsymbol{x}}$. For each linear velocity entry, $\boldsymbol{v}_{i}, G$ has a row of the form $\left[\begin{array}{ll}I_{3} & -\boldsymbol{r}_{i}^{\times}\end{array}\right]$, where $I_{k}$ is the $k \times k$ identity matrix and $\boldsymbol{r}_{i}^{\times}$is the skew-symmetric matrix for the crossproduct of $\boldsymbol{r}_{i}$. For each angular velocity entry, $\boldsymbol{\omega}_{i}$, there is a row of the form $\left[\begin{array}{ll}0 & I_{3}\end{array}\right]$. Similarly, the rows in $\boldsymbol{h}$ compatibly capture the remaining acceleration terms in (3).

\section{Closed-Chain Kinematics}

As discussed earlier, it can be advantageous to specify only a subset of the object DOFs in the primary task, allowing the remaining DOFs to be allocated to the secondary task. Let $\dot{z}$ represent the $p$ DOFs of the object selected, given in terms of a $p \times 6$ selection matrix $S$ as $\dot{z}=S \dot{\boldsymbol{y}}$. If $S$ is constant, then $\ddot{z}=S \ddot{y}$ and

$$
\ddot{\boldsymbol{y}}=S^{+} \ddot{\boldsymbol{z}}+S^{\perp} \boldsymbol{\mu},
$$

where $S^{+}$is the pseudoinverse of $S, S^{\perp}$ is a $6 \times(6-p)$ matrix spanning the null space of $S$, and $\boldsymbol{\mu} \in \mathbb{R}^{6-p}$ is arbitrary. $\boldsymbol{\mu}$ parameterizes the extra DOFs available to the secondary task.

To describe just the constraints that the primary task places on the secondary one, we eliminate $\boldsymbol{\mu}$ as follows. Start by substituting (6) into (5).

$$
J \ddot{\boldsymbol{q}}+\dot{J} \dot{\boldsymbol{q}}=G\left(S^{+} \ddot{\boldsymbol{z}}+S^{\perp} \boldsymbol{\mu}\right)+\boldsymbol{h}
$$

To eliminate $\boldsymbol{\mu}$, we need to find a full-rank matrix $E$ such that:

$$
E G S^{\perp}=0,
$$

where $E \in \mathbb{R}^{(6 n+p-6) \times 6 n}$. Multiplying (7) by $E$ gives the reduced set of equations.

$$
\begin{aligned}
E J \ddot{\boldsymbol{q}}+E \dot{J} \dot{\boldsymbol{q}} & =E G S^{+} \ddot{\boldsymbol{z}}+E \boldsymbol{h} \\
& =E G S^{+} S \ddot{\boldsymbol{y}}+E \boldsymbol{h}
\end{aligned}
$$

Note that $E$ is not unique: any full-rank annihilator of $G S^{\perp}$ will suffice. In general, $E$ may be found by standard linear algebra operations on $G S^{\perp}$, but we may advantageously precompute it for the most common combinations of task type and grasp type. We take up this exercise in the following section.

We see that the matrix EJ plays a similar role in the closed-chain kinematics as the Jacobian matrix usually plays in the open-chain kinematics. Drawing on this analogy, we define the following matrices:

$$
\hat{J} \doteq E J, \quad \hat{\dot{J}} \doteq E \dot{J}, \quad \hat{G} \doteq E G S^{+} S, \quad \hat{\boldsymbol{h}} \doteq E \boldsymbol{h} .
$$

This allows us to define our final closed-chain transformation in the following compact form.

$$
\hat{J} \ddot{\boldsymbol{q}}+\hat{\dot{J}} \dot{\boldsymbol{q}}=\hat{G} \ddot{\boldsymbol{y}}+\hat{\boldsymbol{h}} .
$$

This transformation is not limited to the impedance controller presented here: it is also applicable to other existing cooperative or coordinated controllers. This will allow the other controllers to also free up DOFs from the object space for a secondary task. For first-order controllers, the transformation becomes $\hat{J} \dot{\boldsymbol{q}}=\hat{G} \dot{\boldsymbol{y}}$, where zero relative velocities are assumed.

\section{Explicit SOLUTiOnS}

As described in the previous section, the closed-chain transformation can either be computed online or derived offline. It turns out that the transformation takes on surprisingly simple expressions for some common grasp and task types, expressions that require essentially no extra computation compared to the open-chain formulations. Given the two grasp types described, we will consider the following three task types.

1) Full pose control: $S=I_{6}, \quad S^{+}=I_{6}, \quad S^{\perp}=\emptyset$.

2) Orientation-only control:

$$
S=\left[\begin{array}{ll}
0 & I_{3}
\end{array}\right], \quad S^{+}=\left[\begin{array}{c}
0 \\
I_{3}
\end{array}\right], \quad S^{\perp}=\left[\begin{array}{c}
I_{3} \\
0
\end{array}\right] .
$$

3) Position-only control:

$$
S=\left[\begin{array}{ll}
I_{3} & 0
\end{array}\right], \quad S^{+}=\left[\begin{array}{c}
I_{3} \\
0
\end{array}\right], \quad S^{\perp}=\left[\begin{array}{c}
0 \\
I_{3}
\end{array}\right] .
$$

For full pose control with any grasp type, there are no free object DOFs, and we have simply $E=I_{6}$. The remaining four cases are as follows.

\section{A. Two-hand, Orientation-only}

$$
E=\left[\begin{array}{cccc}
I_{3} & 0 & -I_{3} & 0 \\
0 & I_{3} & 0 & 0 \\
0 & 0 & 0 & I_{3}
\end{array}\right]
$$

B. Two-hand, Position-only

$$
E=\left[\begin{array}{cccc}
I_{3} & \boldsymbol{r}_{1}^{\times} & 0 & 0 \\
0 & 0 & I_{3} & \boldsymbol{r}_{2}^{\times} \\
0 & I_{3} & 0 & -I_{3}
\end{array}\right]
$$

C. Three-finger, Orientation-only

$$
E=\left[\begin{array}{ccc}
I_{3} & -I_{3} & 0 \\
I_{3} & 0 & -I_{3}
\end{array}\right]
$$

\section{Three-finger, Position-only}

This final scenario is more challenging, due to the difficulty of explicitly eliminating the free variable $\dot{\omega}$ from the set of motion constraints. For this scenario,

$$
G S^{\perp}=\left[\begin{array}{c}
-\boldsymbol{r}_{1}^{\times} \\
-\boldsymbol{r}_{2}^{\times} \\
-\boldsymbol{r}_{3}^{\times}
\end{array}\right] .
$$

Since the three contacts are not collinear, we may assume that $\boldsymbol{r}_{1} \times \boldsymbol{r}_{2} \neq 0$ (after possibly renumbering the points). Then, after solving for the scalars $\alpha, \beta, \gamma$ such that,

$$
\boldsymbol{r}_{3}=\alpha \boldsymbol{r}_{1}+\beta \boldsymbol{r}_{2}+\gamma \boldsymbol{r}_{1} \times \boldsymbol{r}_{2} \text {. }
$$

one may take $E$ as

$$
E=\left[\begin{array}{ccc}
\boldsymbol{r}_{1}^{T} & 0 & 0 \\
0 & \boldsymbol{r}_{2}^{T} & 0 \\
\boldsymbol{r}_{2}^{T} & \boldsymbol{r}_{1}^{T} & 0 \\
\alpha I_{3}-\gamma \boldsymbol{r}_{2}^{\times} & \beta I_{3}+\gamma \boldsymbol{r}_{1}^{\times} & -I_{3}
\end{array}\right]
$$


While the derivation of $E$ in this case is not obvious, one may check that it annihilates $G S^{\perp}$ and that it is full rank.

\section{OBJECT DYNAMICS}

Before turning to the control law, we still need to understand the net contribution of the contact forces on the object. This includes both the external dynamics as well as the internal forces acting on the object.

For the external dynamics, consider once again the freebody diagram in Fig. 1. The equation of motion for the object can be expressed as follows.

$$
\begin{gathered}
\boldsymbol{F}_{m a}=\boldsymbol{F}+G^{T} \boldsymbol{f}+m \hat{\boldsymbol{g}} \\
\boldsymbol{F}_{m a} \doteq\left(\begin{array}{c}
m \boldsymbol{a}_{G} \\
I_{G} \dot{\boldsymbol{\omega}}+\boldsymbol{\omega} \times I_{G} \boldsymbol{\omega}+\boldsymbol{r}_{G} \times m \boldsymbol{a}_{G}
\end{array}\right) \\
\hat{\boldsymbol{g}} \doteq\left(\begin{array}{c}
\boldsymbol{g} \\
\boldsymbol{r}_{G} \times \boldsymbol{g}
\end{array}\right)
\end{gathered}
$$

Here, $\boldsymbol{F}_{m a}$ is the inertial forces written in terms of: $m$, the mass of the object; $I_{G}$, the moment of inertia about the center of mass, $G$; $\boldsymbol{a}_{G}$, the acceleration of point $G$; and $\boldsymbol{r}_{G}$, the position vector from the reference point $O$ to $G$. On the right-hand side, $\boldsymbol{f}$ is the column matrix of contact forces, $\boldsymbol{f}_{i}$, and contact torques, $\boldsymbol{t}_{i}$, (see Fig. 1) arranged to mirror the list of velocities, $\boldsymbol{v}_{i}$, and angular velocities, $\boldsymbol{\omega}_{i}$, that appear in $\dot{\boldsymbol{x}}$. Also, $\boldsymbol{F}$ is the net external wrench (force and moment) about point $O$, and $\boldsymbol{g}$ is the gravity vector.

For the internal forces, one can see from (14) that they are defined by the null space of $G^{T}$. Our approach is to use the relative acceleration terms to control the internal forces; hence, they too must lie in the same space. For the sake of this work, we will control the interaction forces between the contacts. An interaction force, $f_{i j}$, is defined as the difference between two contact forces projected along the line between the contact points [10]. It provides a physically relevant parameter, i.e. the squeeze force, that lies in the null space of all grasps. Accordingly, we will use the relative accelerations to close a servo loop about the interaction forces. Consider the example of a two point contact, where $\boldsymbol{u}_{i j}$ is the unit vector pointing from contact point $i$ to $j$.

$$
\begin{aligned}
\boldsymbol{a}_{r e l_{i}}= & \left(k_{P}+k_{I} \int d t\right)\left(f_{i j}^{*}-f_{i j}\right) \boldsymbol{u}_{i j} \\
& f_{i j} \doteq\left(\boldsymbol{f}_{i}-\boldsymbol{f}_{j}\right) \cdot \boldsymbol{u}_{i j}
\end{aligned}
$$

$k_{P}$ and $k_{I}$ are the constant gains.

\section{CONTROL LAW}

Using these impedance tasks, motion transformations, and internal forces, we can now present the control law. First, we will start by modeling the equations of motion for the full system of manipulators.

$$
M \ddot{\boldsymbol{q}}+c-\boldsymbol{\tau}_{f}=\boldsymbol{\tau}
$$

Here, $M$ is the joint space inertia matrix, $c$ is the column matrix of Coriolis, centripetal and gravitational generalized forces, $\boldsymbol{\tau}_{f}$ is the set of joint torques induced by external forces, and $\tau$ is the column matrix of joint torques. Assuming that forces act on the manipulator only at its contact points with the object,

$$
\boldsymbol{\tau}_{f}=-J^{T} \boldsymbol{f}
$$

\section{A. Estimation}

In preparation for the control law, some unsensed quantities for the object need to be estimated. First, the external wrench, $\boldsymbol{F}$, needs to be estimated from the other forces on the object. Referring to (14), we ignore the inertial forces of the object to obtain the quasi-static estimate

$$
\boldsymbol{F}=-G^{T} \boldsymbol{f}-m \hat{\boldsymbol{g}} .
$$

Although included here, the object weight can also be neglected in most cases. In addition, the object velocity can be estimated with the following least-squares error estimate of the system as a rigid body:

$$
\dot{\boldsymbol{y}}=G^{+} J \dot{\boldsymbol{q}}
$$

where the superscript $\left(^{+}\right)$indicates the pseudoinverse of the respective matrix.

\section{B. Inverse Dynamics Controller}

An Inverse Dynamics Controller [9] simply substitutes a commanded joint acceleration, $\ddot{\boldsymbol{q}}^{*}$, for $\ddot{\boldsymbol{q}}$ in (16):

$$
\tau=M \ddot{\boldsymbol{q}}^{*}+\boldsymbol{c}-\boldsymbol{\tau}_{f}
$$

The commanded joint acceleration is expressed in terms of the commanded object acceleration, $\ddot{\boldsymbol{y}}^{*}$, according to (11) as

$$
\begin{gathered}
\ddot{\boldsymbol{q}}^{*}=\hat{J}^{+}\left(\hat{G} \ddot{\boldsymbol{y}}^{*}+\hat{\boldsymbol{h}}-\hat{\dot{J}} \dot{\boldsymbol{q}}\right)+N_{\hat{J}} \ddot{\boldsymbol{q}}_{n s}^{*} \\
N_{\hat{J}} \doteq I-\hat{J}^{+} \hat{J}
\end{gathered}
$$

where $\ddot{\boldsymbol{q}}_{n s}^{*}$ is an arbitrary vector of accelerations, which is projected orthogonally into the null space of $\hat{J}$ via $N_{\hat{J}}$. The two commanded accelerations, $\ddot{\boldsymbol{y}}^{*}$ and $\ddot{\boldsymbol{q}}_{n s}^{*}$, are found from the impedance specifications in (1) and (2):

$$
\begin{aligned}
\ddot{\boldsymbol{y}}^{*} \doteq & M_{o}^{-1}\left(\boldsymbol{F}-\boldsymbol{F}^{*}-B_{o} \dot{\boldsymbol{y}}-K_{o} \Delta \boldsymbol{y}\right), \\
\ddot{\boldsymbol{q}}_{n s}^{*} \doteq & M_{j}^{-1}\left(\boldsymbol{\tau}_{f}-B_{j} \dot{\boldsymbol{q}}-K_{j} \Delta \boldsymbol{q}\right) .
\end{aligned}
$$

The compensation of internal forces on the object occurs as relative acceleration commands calculated from (15) which then plug into the evaluation of $\boldsymbol{h}$.

To understand the true behavior of the control law, consider the closed-loop analysis of the system. The following independent closed-loop dynamics can be derived for both the range space and null space of the system.

$$
\begin{gathered}
S\left[\ddot{\boldsymbol{y}}+M_{o}^{-1}\left(B_{o} \dot{\boldsymbol{y}}+K_{o} \Delta \boldsymbol{y}-\Delta \boldsymbol{F}\right)\right]=S M_{o}^{-1} \boldsymbol{F}_{m a} \\
N_{\hat{J}}\left[\ddot{\boldsymbol{q}}+M_{j}^{-1}\left(B_{j} \dot{\boldsymbol{q}}+K_{j} \Delta \boldsymbol{q}-\boldsymbol{\tau}_{f}\right)\right]=0
\end{gathered}
$$

The first relation reveals the desired object impedance task in (1) applied to the DOFs selected by $S$. If the impedance matrices are diagonal, the task spaces will remain decoupled. The right-hand side of this relation represents a disturbance from the object accelerations due to the quasi-static estimation of $\boldsymbol{F}$. Notably, this disturbance does not effect the internal forces. The second relation shows that the desired 


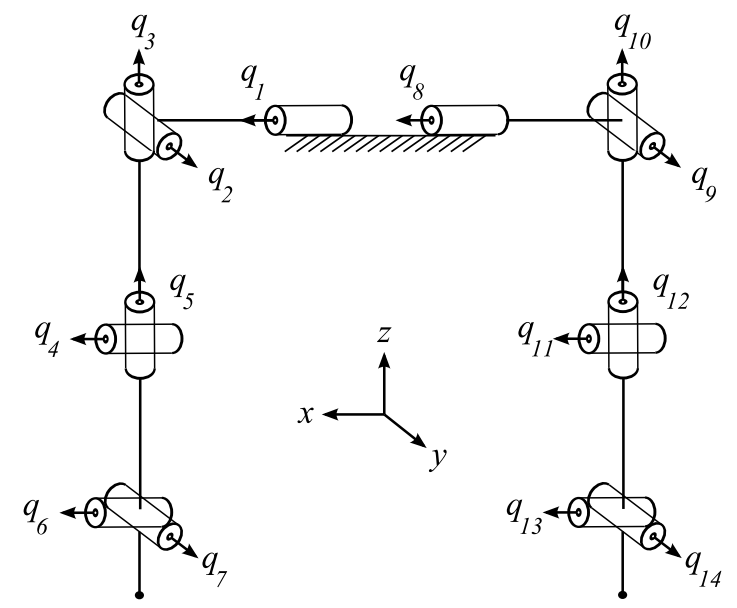

Fig. 2. Kinematic model of the robot. All joint angles are shown in the zero position, and positive rotation is defined in a right-hand sense. The origin is located at the center of the shoulder.

\begin{tabular}{|l|ccc|}
\hline link & $\begin{array}{c}\text { length } \\
(\mathrm{m})\end{array}$ & $\begin{array}{c}\text { mass } \\
(\mathrm{kg})\end{array}$ & $\begin{array}{c}\text { inertia } \\
\left(\mathrm{g} \mathrm{m}^{2}\right)\end{array}$ \\
\hline \hline shoulders & 0.2 & - &,,-- 200 \\
upper arms & 0.4 & 7 & $100,100,1$ \\
lower arms & 0.4 & 7 & $100,100,1$ \\
hands & 0.1 & 2 & $8,8,8$ \\
object & 0.4 & 2 & $8,8,8$ \\
\hline
\end{tabular}

TABLE I

MOdel Properties

secondary impedance task in (2) is implemented with a minimum-error projection into the collective null space. The free DOFs from the object are thus shared amongst the manipulators for the secondary task.

\section{Simulation EXPERIMENTS}

\section{A. Model}

We developed a fully dynamic simulation to test the control law. The simulation consisted of two manipulator arms and a spatial object. The manipulators each modeled a humanoid arm with seven DOF and three links: an upper arm, lower arm, and hand. The kinematics of those arms are shown in Fig. 2. The contact constraints between the end-effectors and the object were enforced through springdamper forces. Each body in the simulation had a symmetric mass distribution, with the center of mass located at the center of the link. The physical properties are listed in Table I. For the six-DOF object, orientation was represented using $x y z$ Euler angles. The orientation error was subsequently converted to an axis-angle representation for the sake of $\Delta y$ in the control law [9].

\section{B. Experiment}

The experiment implemented a two-hand grasp with the position-only task definition. The robot was asked to hold the position of the object fixed while achieving a desired configuration in the joint space. Since the object orientation was left free, the experiment was expected to rotate the object while minimizing the errors in the secondary impedance.
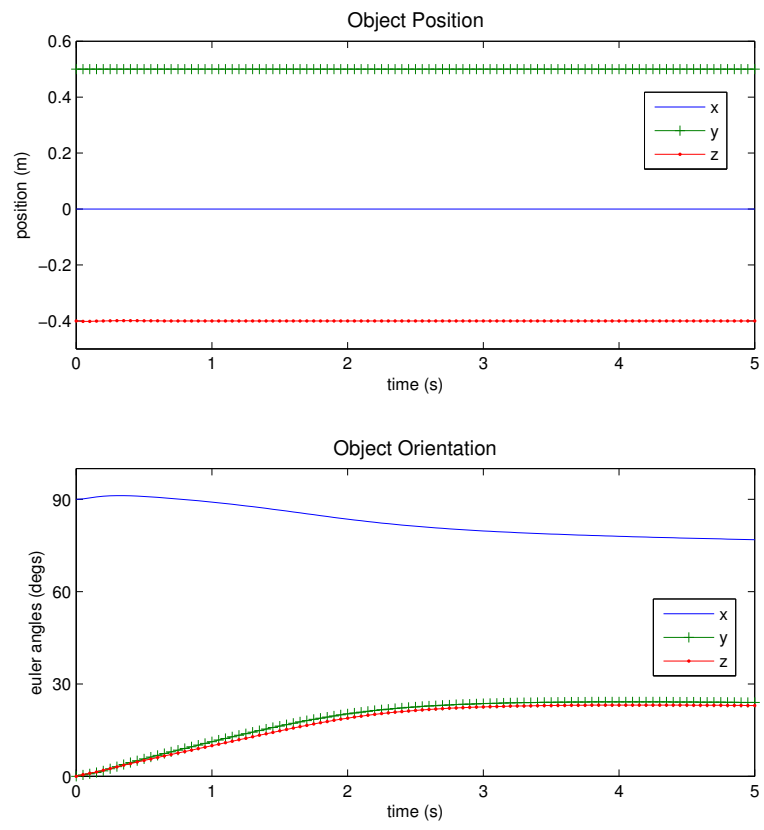

Fig. 3. Object pose for the first experiment. The position remained fixed while the object rotated to minimize the joint space errors.

The robot started out in the initial position shown in Fig. 2-but with the lower arms pointing out horizontally $\left(q_{4}=q_{11}=90^{\circ}\right)$. This placed the object at an initial orientation of $(90,0,0)$ degs and an initial position of $(0,0.5,-0.4)$ $\mathrm{m}$. In this position, we provided a step input to the joint space impedance commanding the right shoulder to swing forward $\left(q_{1}=30^{\circ}\right)$. This command tended to pull the left elbow into the torso, and so we added a second command to keep the left elbow out $\left(q_{9}=-10^{\circ}\right)$. These commands were implemented through the stiffness of the secondary impedance, where the only non-zero elements of $K_{j}$ were the diagonal elements corresponding to $q_{1}$ and $q_{9}$. Hence, all the other joints have no position reference. The damping was tuned for an overdamped response.

The controller demonstrated the expected results. The commanded joints converged stably to their references with no steady-state error. The secondary impedance rotated the object to satisfy its commands, without perturbing the position. The object pose is displayed in Fig. 3, and the joint angles are displayed in Fig. 4. In comparison, using fullpose control, the joints would only have been able to reach a steady-state value of $q_{1}=1^{\circ}$ and $q_{9}=-6^{\circ}$.

In addition, the experiment tested the ability to regulate internal forces. A desired interaction force of $f_{12}^{*}=20 \mathrm{~N}$ was commanded, which equals the weight of the object. Although the robot grasp started out initally with zero interaction force, the controller quickly converged to the reference value and held the value steady throughout the run. Shown in Fig. 5, the interaction force is unperturbed by the object accelerations, although these accelerations are not fed back. The complete list of controller parameters is available in Table II. 

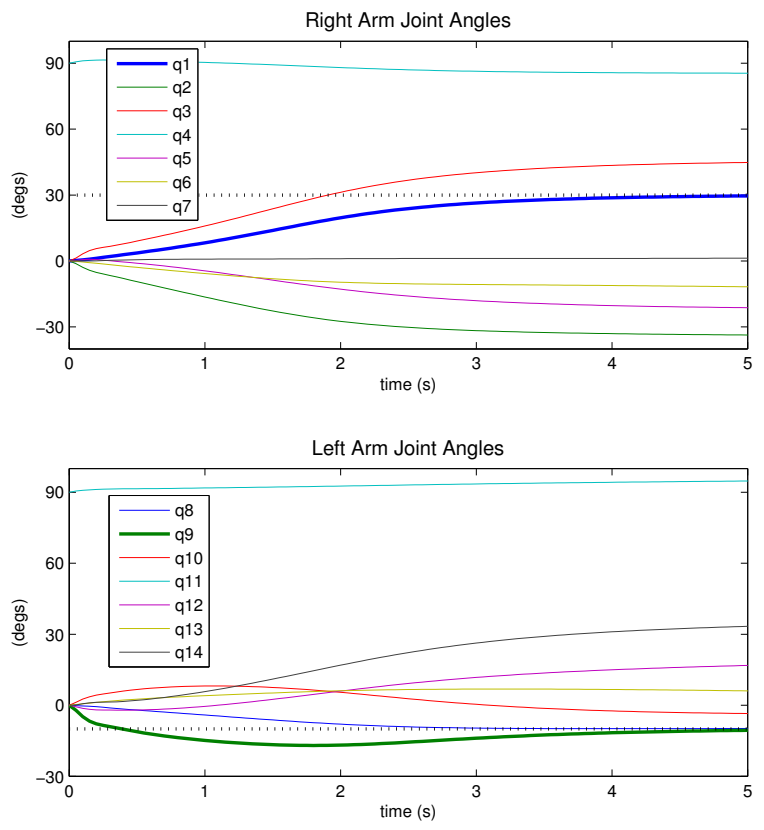

Fig. 4. Joint values for the first experiment. The commanded joints (bold lines) successfully converged to the reference values (dotted lines).

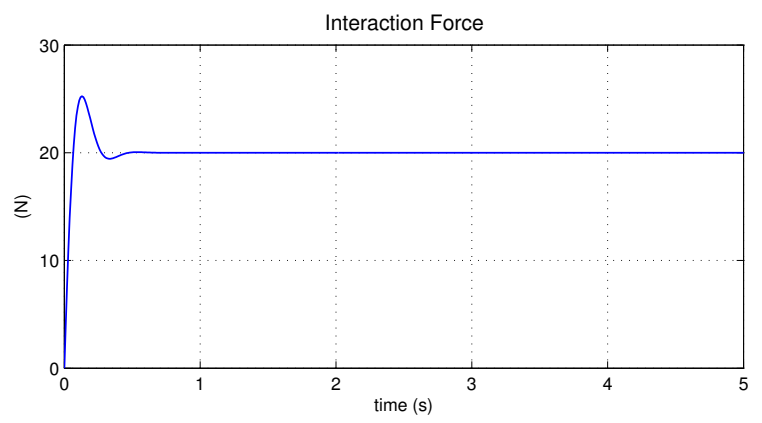

Fig. 5. The controller successfully maintained the desired interaction force between both hands, unperturbed by either object or joint space motions.

Throughout the experiments, the effectiveness of the task hierarchy was demonstrated. The secondary impedance consistently operated in the null space without interfering with either the object impedance nor the object internal forces. Other experiments showed the controller moving the object with the desired impedance response, while controlling the joints in an orthogonal space.

\begin{tabular}{|l|c||l|c|}
\hline parameter & value & parameter & value \\
\hline \hline$M_{O}$ & $I_{6}$ & $M_{j}$ & $M$ \\
$B_{O}$ & {$\left[\begin{array}{cc}30 I_{3} & 0 \\
0 & 3 I_{3} \\
200 I_{3} & 0 \\
0 & 5 I_{3}\end{array}\right]$} & $B_{j}$ & $20 I_{6}$ \\
$K_{O}$ & $K_{j_{11}}, K_{j_{99}}$ & 600 \\
$k_{P}$ & $k_{I}$ & 0 \\
\hline
\end{tabular}

TABLE II

CONTROLler Parameters.

\section{DISCUSSION}

The framework of a primary object impedance and secondary joint impedance offers substantial advantages for humanoid robots performing assembly tasks. The impedance formulation inherently lends itself to robust interaction with the environment with flexible objectives. The presence of the joint space impedance adds the ability to achieve such utilitarian objectives as obstacle avoidance, joint limit avoidance, and null space damping. The hierarchy then allows these joint level commands to be applied without compromising the object behavior or grasp. This can be an important safety feature when the object is engaged with the environment. The combination of these features thus forms a nice framework for cooperative assembly tasks.

A significant contribution in this work is the ability to select a subset of the object's degrees of freedom in the primary task, allowing the remaining object DOFs to assist in the attainment of a secondary task. We formulate this in terms of a closed-chain Jacobian and grasp matrix, $\hat{J}$ and $\hat{G}$ respectively. A compatible control law is proposed and validated in simulations of a two-armed robot. The closedchain Jacobian and grasp matrices are not limited to this control law; they can be applied to other existing cooperative controllers, be they motion- or force-based controllers.

\section{REFERENCES}

[1] N. Hogan, "Impedance control: An approach to manipulation I-III," Journal of Dynamic Systems, Measurement, and Control, vol. 107, pp. $1-24,1987$.

[2] Y. Nakamura, Advanced Robotics: Redundancy and Optimization. Addison-Wesley Publishing, 1991.

[3] R. Platt, M. Abdallah, and C. Wampler, "Multi-priority cartesian impedance control," in Proceedings of Robotics: Science and Systems Conference (RSS), Zaragoza, Spain, June 2010.

[4] M. Koga, K. Kosuge, K. Furuta, and K. Nosaki, "Coordinated motion control of robot arms based on the virtual internal model," IEEE Trans. on Robotics and Automation, vol. 8, no. 1, pp. 77-85, February 1992.

[5] T. Wimbock, C. Ott, and G. Hirzinger, "Impedance behaviors for twohanded manipulation: Design and experiments," in IEEE International Conference on Robotics and Automations (ICRA), April 2007, pp. 4182-4189.

[6] S. Schneider and R. Cannon, "Object impedance control for cooperative manipulation: Theory and experimental results," IEEE Transactions on Robotics and Automation, vol. 8, no. 3, 1992.

[7] R. B. Bonitz and T. C. Hsia, "Internal force-based impedance control for cooperating manipulators," IEEE Trans. on Robotics and Automation, vol. 12, no. 1, February 1996.

[8] F. Caccavale, P. Chiacchio, A. Marino, and L. Villani, "SixDOF impedance control of dual-arm cooperative manipulators," IEEE/ASME Trans. on Mechatronics, vol. 13, pp. 576-586, Oct. 2008.

[9] M. W. Spong, S. Hutchinson, and M. Vidyasagar, Robot Modeling and Control. John Wiley \& Sons, 2006.

[10] V. Kumar and K. Waldron, "Force distribution in closed kinematic chains," IEEE Journal of Robotics and Automation, vol. 4, no. 6, pp. 657-664, December 1988. 